Supporting Information

\title{
An ex vivo model enables systematic investigation of the intestinal absorption and transcytosis of oral particulate
}

\section{nanocarriers}

Zhengyang Jia, Anthony Wignall, Ludivine Delon, Zhaobin Guo, Clive Prestidge, and Benjamin

Thierry*

Z. Jia, L. Delon, Z. Guo, Prof. B. Thierry

Future Industries Institute and ARC Centre of Excellence Convergent Bio-Nano Science and Technology, University of South Australia, Mawson Lakes Campus, Adelaide, SA, 5095, Australia

A. Wignall, Prof. C. Prestidge

UniSA Clinical and Health Science and ARC Centre of Excellence Convergent Bio-Nano Science and Technology, University of South Australia, City West Campus, Adelaide, SA, 5000, Australia

Corresponding Author: Benjamin Thierry

E-mail: benjamin.thierry@unisa.edu.au 

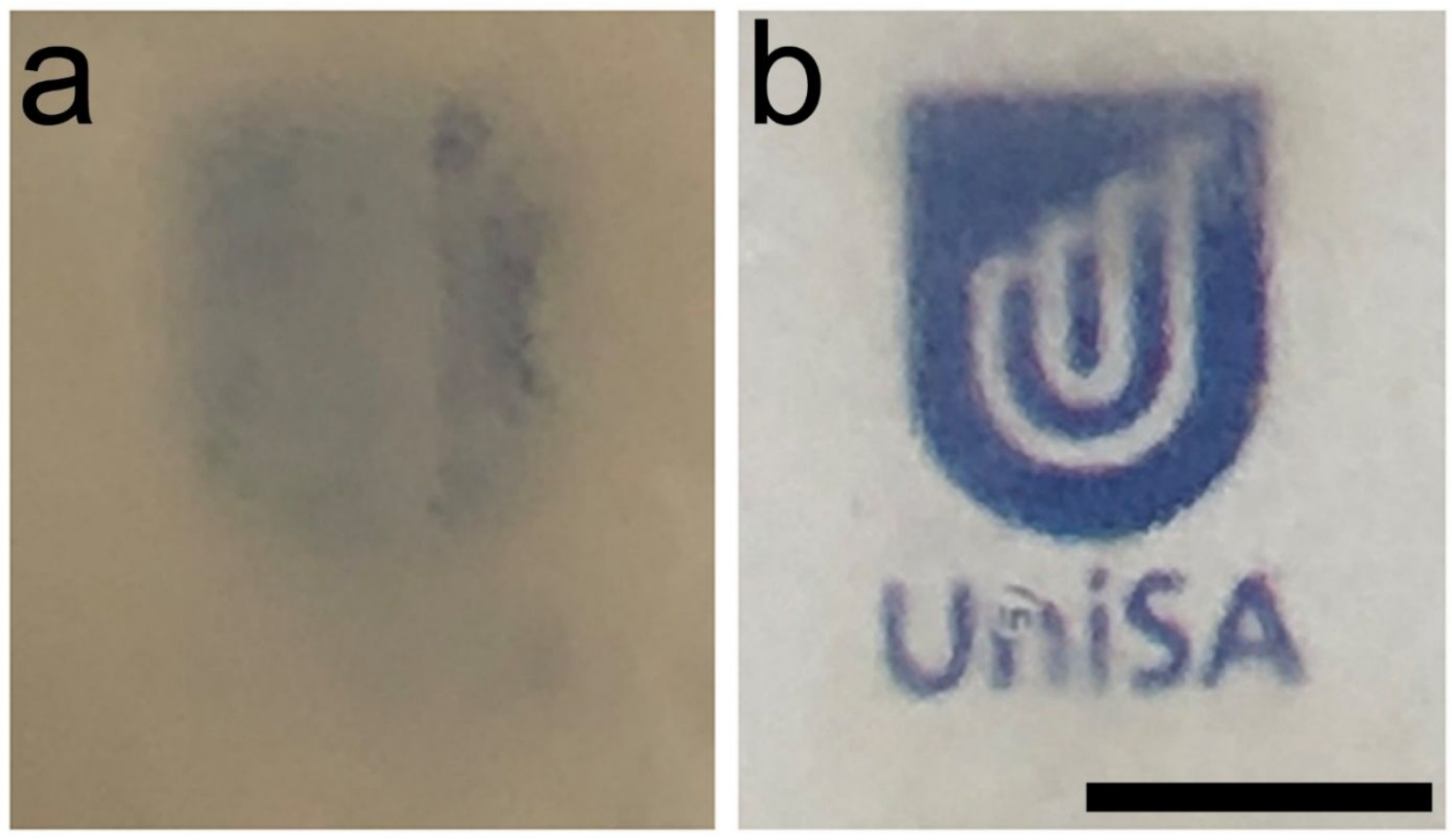

Figure S1. Photos of the intestinal tissues (a) before and (b) after tissue clearing treatment. The logo of the authors' affiliation beneath the cleared intestinal tissue becomes visible. Scale bar: 0.5 cm.
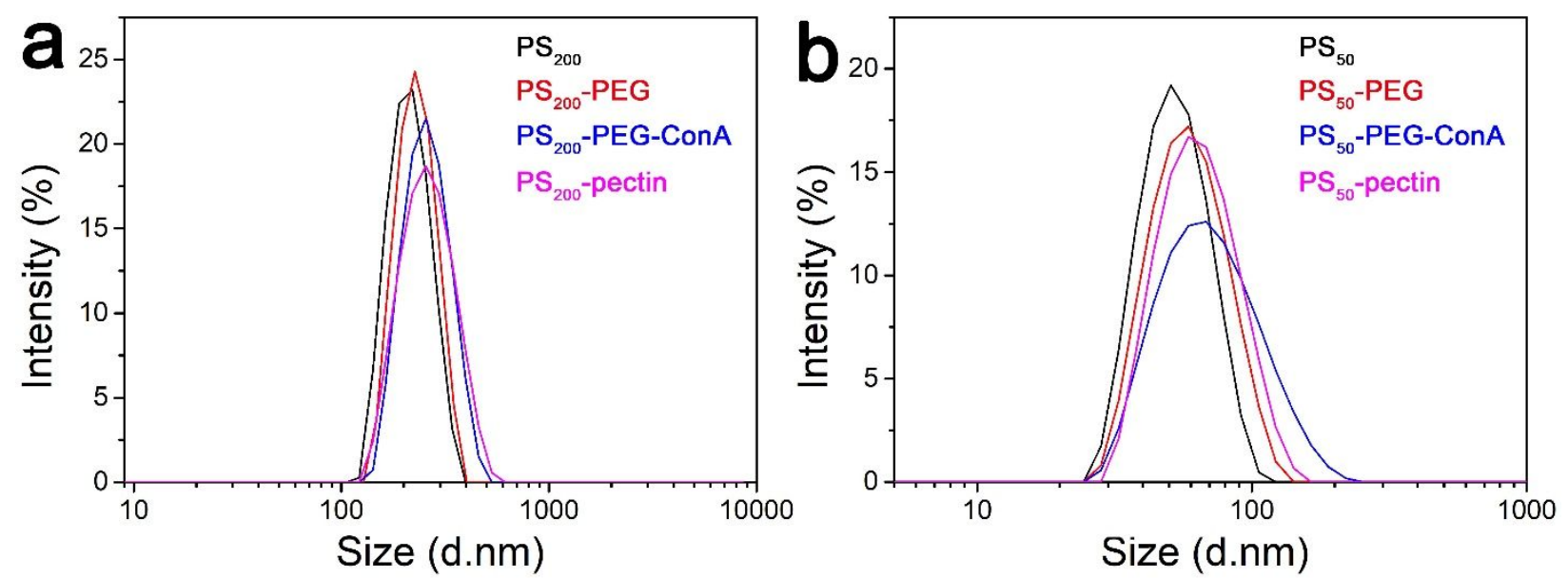

Figure S2. DLS measurements display the hydrodynamic diameter distributions of the model nanoparticles 

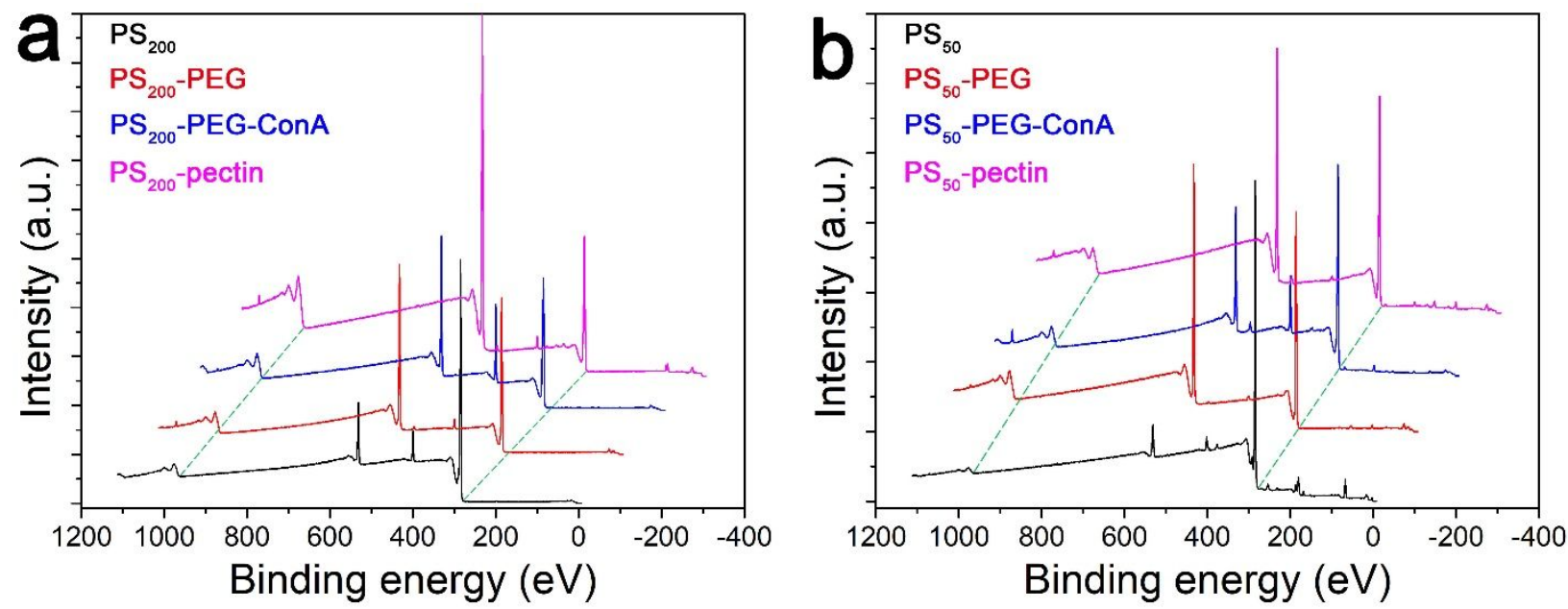

Figure S3. XPS elemental survey spectra of (a) $\mathrm{PS}_{200}, \mathrm{PS}_{200^{-}} \mathrm{PEG}, \mathrm{PS}_{200^{-}} \mathrm{PEG}-\mathrm{ConA}$ and $\mathrm{PS}_{200^{-}}$ pectin; (b) $\mathrm{PS}_{50}, \mathrm{PS}_{50}$-PEG, $\mathrm{PS}_{50}$-PEG-ConA and $\mathrm{PS}_{50}$-pectin
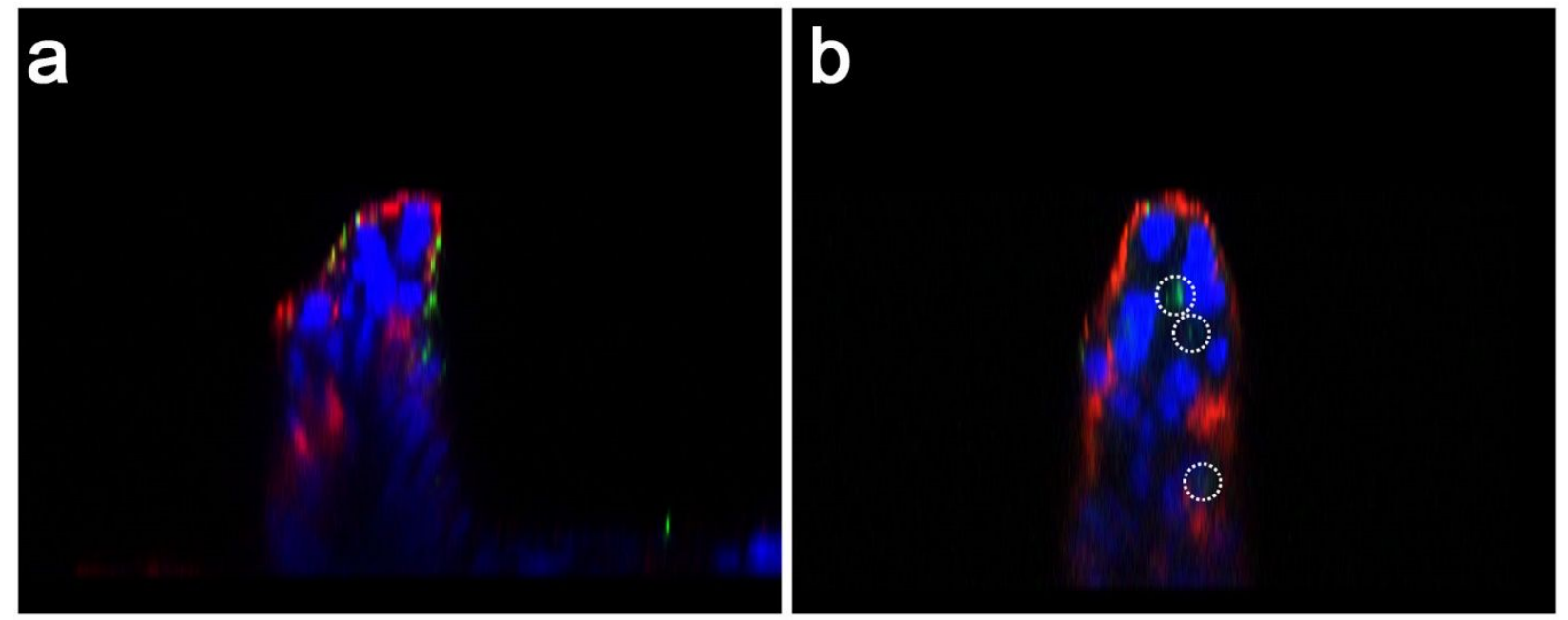

Figure S4. Side plane view of a single intestinal villus after incubation with (a) $\mathrm{PS}_{200}$-PEG-ConA and (b) $\mathrm{PS}_{50}$-PEG-ConA for $2 \mathrm{~h}$. Nanoparticle aggregates in villous core are indicated by the white dashed circles. 
